\title{
Computing Tropical Points and Tropical Links
}

\author{
Tommy Hofmann ${ }^{1} \cdot$ Yue Ren ${ }^{2}$ (])
}

Received: 15 December 2016 / Revised: 21 March 2018 / Accepted: 13 July 2018 /

Published online: 7 August 2018

(c) The Author(s) 2018

\begin{abstract}
We present an algorithm for computing zero-dimensional tropical varieties based on triangular decomposition and Newton polygon methods. From it, we derive algorithms for computing points on and links of higher-dimensional tropical varieties, using intersections with affine hyperplanes to reduce the dimension to zero. We use the algorithms to show that the tropical Grassmannians $\mathcal{G}_{3,8}$ and $\mathcal{G}_{4,8}$ are not simplicial.
\end{abstract}

Keywords Tropical geometry · Tropical variety · Tropical Grassmannian · Computer algebra $\cdot$ Newton polygon

Mathematics Subject Classification $14 \mathrm{~T} 05 \cdot 52 \mathrm{~B} 20 \cdot 12 \mathrm{~J} 25 \cdot 13 \mathrm{P} 15$

\section{Introduction}

Given an affine variety $X$ over an algebraically closed field $K$ with non-trivial valuation, its tropical variety $\operatorname{Trop}(X)$ is the Euclidean closure of its image under component-wise valuation. Tropical varieties arise naturally in many applications in mathematics $[1,22]$ and beyond, such as in the context of phylogenetic trees in biol-

Editor in Charge: Kenneth Clarkson

The second author was partially supported by the DFG Priority Programme 1489 "Algorithmic and Experimental Methods in Algebra, Geometry and Number Theory" and the Center of Advanced Studies in Mathematics of Ben-Gurion University. This work was completed during the program "Tropical Geometry, Amoebas and Polytopes" at the Institute Mittag-Leffler. The second author would like to thank the institute for its hospitality.

Tommy Hofmann

thofmann@mathematik.uni-kl.de

Yue Ren

yueren@mis.mpg.de

1 Technische Universität Kaiserslautern, 67653 Kaiserslautern, Germany

2 Max-Planck-Institut für Mathematik in den Naturwissenschaften, 04103 Leipzig, Germany 
ogy [26, Sect. 4], product-mix auctions in economics [27] or finiteness of central configurations in the 5-body problem in physics [10].

Nevertheless, computing tropical varieties is an algorithmically challenging task, requiring sophisticated techniques from computer algebra and convex geometry. The first algorithms were developed by Bogart et al. [2] for the field of complex Puiseux series $\mathbb{C}\{\{t\}\}$. More recently, Chan and Maclagan introduced a new notion of Gröbner bases for general fields with valuation in order to compute tropical varieties thereover [4]. Concurrently, Chan developed a special algorithm for computing tropical curves [3, Chap. 4]. All these algorithms have been implemented in GFAN [15], which is the currently most widely used program for computing tropical varieties. In this article, we touch upon two problems that arise in the computation.

The first problem is to pinpoint a tropical starting point, a first point on the tropical variety from which all further computations start off. At present, the default is to traverse the Gröbner complex randomly while checking all vertices along the way for containment in the tropical variety. This is a rather inefficient approach however, as there can be significantly more Gröbner polyhedra outside the tropical variety than inside [2, Thm. 6.3]. The second problem, which arises repeatedly, is to compute tropical links, tropical varieties of simpler combinatorial structure which describe the original tropical variety locally. Their special structure allows them to be computed via tropical prevarieties. While this has proven to be successful for a wide range of examples, experiments show that with increasing input size the tropical prevariety computations become intractable.

We present a simple yet novel approach for solving the aforementioned problems, based on the following bread-and-butter techniques in computer algebra and number theory:

1. intersection with random hyperplanes,

2. triangular decomposition of zero-dimensional polynomial ideals,

3. reading off valuations of roots from Newton polygons.

Moreover, the algorithm for tropical links also relies on a generalization of the Transverse Intersection Lemma [2, Lem. 3.2] to general fields with valuation, which follows from recent results by Osserman and Payne [24].

We use our algorithms to study some higher tropical Grassmannians $\mathcal{G}_{k, n}$. They were first studied by Speyer and Sturmfels [25], who showed that $\mathcal{G}_{2, n}$ for $n \geq 2$ and $\mathcal{G}_{3,6}$ are simplicial fans, the former using an intriguing connection to spaces of phylogenetic trees and the latter through explicit computation. Additionally, in their work on the parametrization and realizability of tropical planes [12], Hermannn, Jensen, Joswig and Sturmfels showed that $\mathcal{G}_{3,7}$ is also a simplicial fan. We will complement these findings by showing that this does not hold for $\mathcal{G}_{3,8}$ and $\mathcal{G}_{4,8}$.

All algorithms presented in this article have been implemented in the SINGULAR library tropicalNewton.1ib [6,14], and are publicly available as part of the official SINGULAR distribution. For computations in convex geometry, it relies on an interface to GFANLIB $[15,16]$.

Convention 1.1 For the remainder of the article, let $K$ be an algebraically closed field with non-trivial valuation $v: K \rightarrow \mathbb{R} \cup\{\infty\}$, though we will mainly focus on its restriction $v: K^{*} \rightarrow \mathbb{R}$. We assume that $1 \in v\left(K^{*}\right)$. As $K$ is algebraically closed, 
there exists a homomorphism $\psi:\left(v\left(K^{*}\right),+\right) \rightarrow\left(K^{*}, \cdot\right)$ with $v(\psi(w))=w[21$, Lem. 2.1.15]. We will fix one such $\psi$ and use $p^{w}$ to denote the element $\psi(w) \in K^{*}$, or $t^{w}$ if $K$ is the field of Puiseux series $\mathbb{C}\{\{t\}$. Let $\mathfrak{K}$ denote the residue field of $K$.

Furthermore, we fix a multivariate polynomial ring $K[x]:=K\left[x_{1}, \ldots, x_{n}\right]$. By abuse of notation, we will also use $v$ to refer to the component-wise valuation $\left(K^{*}\right)^{n} \rightarrow \mathbb{R}^{n}$.

\section{Computing zero-dimensional tropical varieties}

In this section we present an algorithm, Algorithm 2.10, for computing zerodimensional tropical varieties using triangular decomposition and Newton polygon methods. For the sake of simplicity, we restrict ourselves to the task of computing a single point on the tropical variety, as the structure of the algorithm easily suggests how the entire tropical variety can be computed with proper bookkeeping. We conclude the section by showing that any generic triangular set admits what we call a tree of unique Newton polygons, which is the best case for our algorithm as it allows us to compute its tropical variety purely combinatorially, see Example 2.13.

Definition 2.1 Let $w \in \mathbb{R}^{n}$. For a polynomial $f=\sum_{\alpha \in \mathbb{N}^{n}} c_{\alpha} \cdot x^{\alpha} \in K[x]$, we define the evaluation of its tropicalization at $w$ to be

$$
\operatorname{trop}(f)(w):=\min \left\{w \cdot \alpha+v\left(c_{\alpha}\right) \mid c_{\alpha} \neq 0\right\}
$$

and its initial form with respect to $w$ to be

$$
\operatorname{in}_{w}(f)=\sum_{w \cdot \alpha+v\left(c_{\alpha}\right)=\operatorname{trop}(f)(w)} \overline{c_{\alpha} p^{-v\left(c_{\alpha}\right)}} \cdot x^{\alpha} \in \mathfrak{K}[x] .
$$

For an ideal $I \unlhd K[x]$, we define its initial ideal with respect to $w$ to be

$$
\operatorname{in}_{w}(I)=\left\langle\operatorname{in}_{w}(f) \mid f \in I\right\rangle \unlhd \mathfrak{K}[x] .
$$

The tropical variety of $I$ is then given by

$$
\operatorname{Trop}(I):=\left\{w \in \mathbb{R}^{n} \mid \operatorname{in}_{w}(I) \text { monomial free }\right\}
$$

For single polynomials $f \in K[x]$ and finite subsets $F \subseteq K[x]$, we abbreviate $\operatorname{Trop}(f):=\operatorname{Trop}(\langle f\rangle)$ and $\operatorname{Trop}(F):=\operatorname{Trop}(\langle F\rangle)$.

The tropical variety is naturally covered by Gröbner polyhedra and hence the support of a subcomplex of the Gröbner complex [21, Thm. 3.3.2]. Its dimension resp. lineality space is the dimension resp. lineality space of that subcomplex.

While the previous algorithms mainly work with the aforementioned definition of tropical varieties, the algorithms in this article focus on the following characterization: 
Theorem 2.2 ([21, Thm. 3.2.5]) For any ideal $I \unlhd K[x]$ and its corresponding affine variety $X=V(I) \subseteq K^{n}$ we have

$$
\operatorname{Trop}(I)=\overline{v\left(X \cap\left(K^{*}\right)^{n}\right)}
$$

where $\overline{(\cdot)}$ denotes the closure in the Euclidean topology.

We now describe how to exploit this geometric characterization algorithmically using triangular sets and Newton polygon methods.

Definition 2.3 A set $F=\left\{f_{1}, \ldots, f_{n}\right\} \subseteq K[x]$ is called triangular, if for each $k=$ $1, \ldots, n$ we have $f_{k} \in K\left[x_{1}, \ldots, x_{k}\right]$ of the form

$$
f_{k}=c_{k} x_{k}^{d_{k}}+\text { terms of lower } x_{k} \text {-degree }
$$

for some $c_{k} \in K^{*}$ and $d_{k} \in \mathbb{N}_{>0}$.

Proposition 2.4 ([9, Cor. 4.7.4]) Let I be a zero-dimensional ideal, then there exist triangular sets $F_{1}, \ldots, F_{s}$ such that

$$
\sqrt{I}=\bigcap_{i=1}^{s} \sqrt{\left\langle F_{i}\right\rangle} \text { and }\left\langle F_{i}\right\rangle+\left\langle F_{j}\right\rangle=\langle 1\rangle \text { for } i \neq j .
$$

Remark 2.5 Triangular decompositions as in Proposition 2.4 were initially introduced by Lazard [20] for polynomial system solving. They are a weaker notion of a primary decomposition and can be obtained easier through various methods, see [20, Procedure 1] or [9, Algorithm 4.7.8] for details.

Definition 2.6 For a univariate polynomial $f=\sum_{i=0}^{d} c_{i} \cdot x_{k}^{i} \in K\left[x_{k}\right], c_{i} \in K$, the Newton polygon or extended Newton polyhedron is defined to be

$$
\Delta(f):=\operatorname{Conv}\left(\left\{\left(i, v\left(c_{i}\right)\right) \mid c_{i} \neq 0\right\}\right)+\left(\{0\} \times \mathbb{R}_{\geq 0}\right) .
$$

Similarly, for a multivariate polynomial $f=\sum_{i=0}^{d} f_{i} \cdot x_{k}^{i} \in K\left[x_{1}, \ldots, x_{k}\right], f_{i} \in$ $K\left[x_{1}, \ldots, x_{k-1}\right]$, and a weight $w \in \mathbb{R}^{k-1}$, we define the expected Newton polygon of $f$ at $w$ to be

$$
\Delta_{w}(f):=\operatorname{Conv}\left(\left\{\left(i, \operatorname{trop}\left(f_{i}\right)(w)\right) \mid f_{i} \neq 0\right\}\right)+\left(\{0\} \times \mathbb{R}_{\geq 0}\right) .
$$

We say $f$ has a unique Newton polygon at $w$, if the initial form $\operatorname{in}_{w}\left(f_{i}\right)$ is a monomial for all vertices $\left(i, \operatorname{trop}\left(f_{i}\right)(w)\right) \in \Delta_{w}(f)$. Let $\Lambda(f)$ resp. $\Lambda_{w}(f)$ denote the sets consisting of the negatives of the slopes of $\Delta(f)$ resp. $\Delta_{w}(f)$.

The following two propositions justify the utility of Newton polygons and the term "unique Newton polygon".

Proposition 2.7 ([23, Prop. II.6.3]) Let $f$ be a univariate polynomial over $K$. Then $\Lambda(f)=\operatorname{Trop}(f)$. 


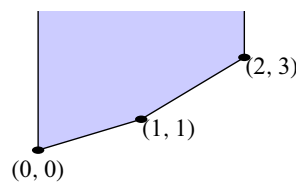

$\Delta\left(f\left(1,3, x_{3}\right)\right)$
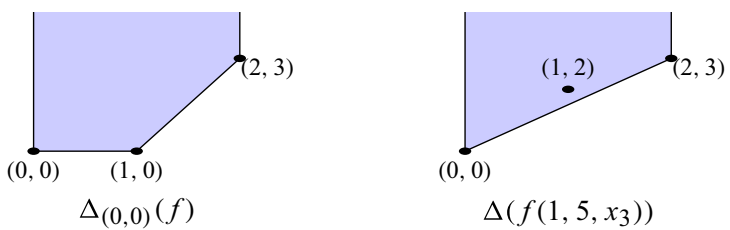

$\Delta\left(f\left(1,5, x_{3}\right)\right)$

Fig. 1 The expected and possible Newton polygons of $f$

Proposition 2.8 For a polynomial $f \in K\left[x_{1}, \ldots, x_{k}\right]$ and a weight $w \in \mathbb{R}^{k-1}$ the following are equivalent:

1. $f$ has a unique Newton polygon at $w$,

2. for all $z \in K^{k-1}$ with $v(z)=w$ we have $\Delta\left(f\left(z, x_{k}\right)\right)=\Delta_{w}(f)$.

Proof Note that for any coefficient $c \in K$, any substitute $z \in K^{k-1}$ with $v(z)=w$ and any exponent vector $\alpha \in \mathbb{N}^{k-1}$ we have $v\left(c \cdot z^{\alpha}\right)=w \cdot \alpha+v(c)=\operatorname{trop}\left(c \cdot x^{\alpha}\right)(w)$. Hence for any $f_{i} \in K\left[x_{1}, \ldots, x_{k-1}\right]$ we always have

$$
v\left(f_{i}(z)\right) \geq \operatorname{trop}\left(f_{i}\right)(w),
$$

with equality guaranteed if $\operatorname{in}_{w}\left(f_{i}\right)$ is a monomial, i.e., (1) implies (2).

For the converse, it suffices to show that the equality is guaranteed only if $f_{i}$ is a monomial. Since $K$ is algebraically closed, so is its residue field $\mathfrak{K}$. In particular, if $\operatorname{in}_{w}\left(f_{i}\right)$ is no monomial, then it has a non-zero root in $\mathfrak{K}^{k-1}$. Picking any $z \in K^{k-1}$ with $v(z)=w$ and $\operatorname{in}_{w}(f)\left(\overline{z_{1} \cdot p^{-v\left(z_{1}\right)}}, \ldots, \overline{z_{k-1} \cdot p^{-v\left(z_{k-1}\right)}}\right)=0, p \in K$, denoting a uniformizing parameter, yields $v\left(f_{i}(z)\right) \gtrless \operatorname{trop}\left(f_{i}\right)(w)$.

Example 2.9 Let $K=\overline{\mathbb{Q}}_{2}$ be the algebraic closure of the 2-adic numbers. The polynomial $f=2^{3} x_{3}^{2}+\left(x_{1}-x_{2}\right) x_{3}+\left(x_{1}^{2}-2 x_{2}\right) \in K[x]$ has a unique Newton polygon at all $\left(w_{1}, w_{2}\right) \in \mathbb{R}^{2}$ with $w_{1} \neq w_{2}$ and $2 w_{1} \neq w_{2}+1$ :

For instance, given $\left(z_{1}, z_{2}\right) \in K^{2}$ with $v_{2}\left(z_{1}, z_{2}\right)=(2,1)$, the Newton polygon $\Delta\left(f\left(z_{1}, z_{2}, x_{3}\right)\right)$ will have vertices at $(0,2),(1,1)$ and $(2,3)$. Using Proposition 2.7 we conclude that $\operatorname{Trop}\left(f\left(z_{1}, z_{2}, x_{3}\right)\right)=\{0,1\}$ and hence $(2,1,0),(2,1,1) \in \operatorname{Trop}(f)$. On the other hand, for $\left(z_{1}, z_{2}\right) \in K^{2}$ with $v_{2}\left(z_{1}, z_{2}\right)=(0,0)$, the Newton polygon $\Delta\left(f\left(z_{1}, z_{2}, x_{3}\right)\right)$ may vary depending on the choice of $z_{1}, z_{2}$, as illustrated in Fig. 1 .

\section{Algorithm 2.10 (Tropical point, zero-dimensional case only)}

Input: $F=\left\{f_{1}, \ldots, f_{n}\right\} \subseteq K[x]$ a triangular set with $V(F) \subseteq\left(K^{*}\right)^{n}$.

Output: $w \in \operatorname{Trop}(F)$.

1: Pick $w_{1} \in \Lambda\left(f_{1}\right)$.

2: for $i=2, \ldots, n$ do

3: if $f_{i}$ has a unique Newton polygon at $\left(w_{1}, \ldots, w_{i-1}\right)$ then

4: $\quad$ Pick $w_{i} \in \Lambda_{\left(w_{1}, \ldots, w_{i-1}\right)}\left(f_{i}\right)$.

5: else

6: $\quad$ Compute a root $\left(z_{1}, \ldots, z_{i-1}\right) \in V\left(f_{1}, \ldots, f_{i-1}\right)$.

7: $\quad$ Pick $w_{i} \in \Lambda\left(f_{i}\left(z_{1}, \ldots, z_{i-1}, x_{i}\right)\right)$.

8: return $\left(w_{1}, \ldots, w_{n}\right)$ 
Proof The termination of the algorithm is clear and the correctness follows directly from Propositions 2.7 and 2.8.

While Algorithm 2.10 looks straightforward, performing Step 6 is a rather delicate task, which we will address in Examples 2.11 and 2.12. Example 2.13 shows how Algorithm 2.10 can be used to compute the entire tropical variety.

Example 2.11 (Root approximation) Note that, in Step 6 of Algorithm 2.10, it always suffices to approximate the root with respect to the metric induced by the valuation. For instance, consider the triangular set $F=\left\{f_{1}, f_{2}, f_{3}\right\} \subseteq \overline{\mathbb{Q}}_{3}\left[x_{1}, x_{2}, x_{3}\right]$ with

$$
f_{1}=x_{1}^{2}+3 x_{1}-1, \quad f_{2}=x_{2}^{2}+9 x_{2}-1, \quad f_{3}=3 x_{3}^{2}+\left(x_{1}-x_{2}\right) x_{3}+1 .
$$

From the Newton polygons of $f_{1}$ and $f_{2}$ we see that elements $\left(z_{1}, z_{2}\right) \in\left(\overline{\mathbb{Q}}_{3}\right)^{2}$ with $f_{1}\left(z_{1}\right)=f_{2}\left(z_{1}, z_{2}\right)=0$ must satisfy $v_{3}\left(z_{1}, z_{2}\right)=(0,0)$. However, $f_{3}$ does not have a unique Newton polygon at $(0,0)$ and $\Delta\left(f_{3}\left(z_{1}, z_{2}, x_{3}\right)\right)$ may vary depending on $z_{1}$ and $z_{2}$. More precisely, we have

$$
\Delta\left(f_{3}\left(z_{1}, z_{2}, x_{3}\right)\right)= \begin{cases}\underbrace{(2,1)}_{(0,0)} & \text { if } v_{3}\left(z_{1}-z_{2}\right)=0, \\ \underbrace{}_{(0,0)} & \text { if } v_{3}\left(z_{1}-z_{2}\right)>0 .\end{cases}
$$

Through Hensel Lifting we see that $f_{1}$ has a root $z_{1} \in \mathbb{Z}_{3}$ with $z_{1} \equiv 4 \bmod 3^{2} \mathbb{Z}_{3}$ and $f_{2}\left(z_{1}, x_{2}\right)$ has a root $z_{2} \in \mathbb{Z}_{3}$ with $z_{2} \equiv 1 \bmod 3^{2} \mathbb{Z}_{3}$. Since $z_{1}-z_{2} \neq 0$ and $z_{1}-z_{2} \in 3 \mathbb{Z}_{3}$, we are in the second case and conclude that $\left(0,0,-\frac{1}{2}\right) \in \operatorname{Trop}(F)$.

Example 2.12 (Field extensions) While we began this article by fixing an algebraically closed field $K$, in practise we are always working over a finite extension of either the rationals $\mathbb{Q}$, a finite field $\mathbb{F}_{q}$ or function fields thereon. This can be problematic in conjunction with Step 6, as approximating roots might require further field extensions. By the recursive nature of the algorithm, we potentially end up with a tower of field extensions. For instance, consider the triangular set $F=\left\{f_{1}, \ldots, f_{n}\right\} \subseteq \mathbb{Q}((t))\left[x_{1}, \ldots, x_{n}\right]$ given by

$$
f_{k}=x_{k}^{2}-q_{k} t\left(\sum_{i=1}^{k-1} x_{i}\right)+q_{k} t^{2}, \text { where } q_{k} \in \mathbb{N} \text { is the } k \text {-th prime. }
$$

This triangular set will never encounter a unique Newton polygon in Step 3, and every root computation in Step 6 will require a new degree 2 extension, as $V\left(f_{1}, \ldots, f_{k}\right) \subseteq$ $\left(\mathbb{Q}\left(\sqrt{q_{1}}, \ldots, \sqrt{q_{k}}\right)\{\{t\}\}\right)^{n} \backslash\left(\mathbb{Q}\left(\sqrt{q_{1}}, \ldots, \sqrt{q_{k-1}}\right)\{\{t\}\}\right)^{n}$. This eventually leads to a degree $2^{n}$ extension of $\mathbb{Q}$, which shows in the performance of our implementation of Algorithm 2.10 in tropicalNewton. lib: computing the tropicalization for $n=13$ requires 8 seconds and it roughly doubles with each increment of $n$. See Timings 3.9 for a comparison with other algorithms. 


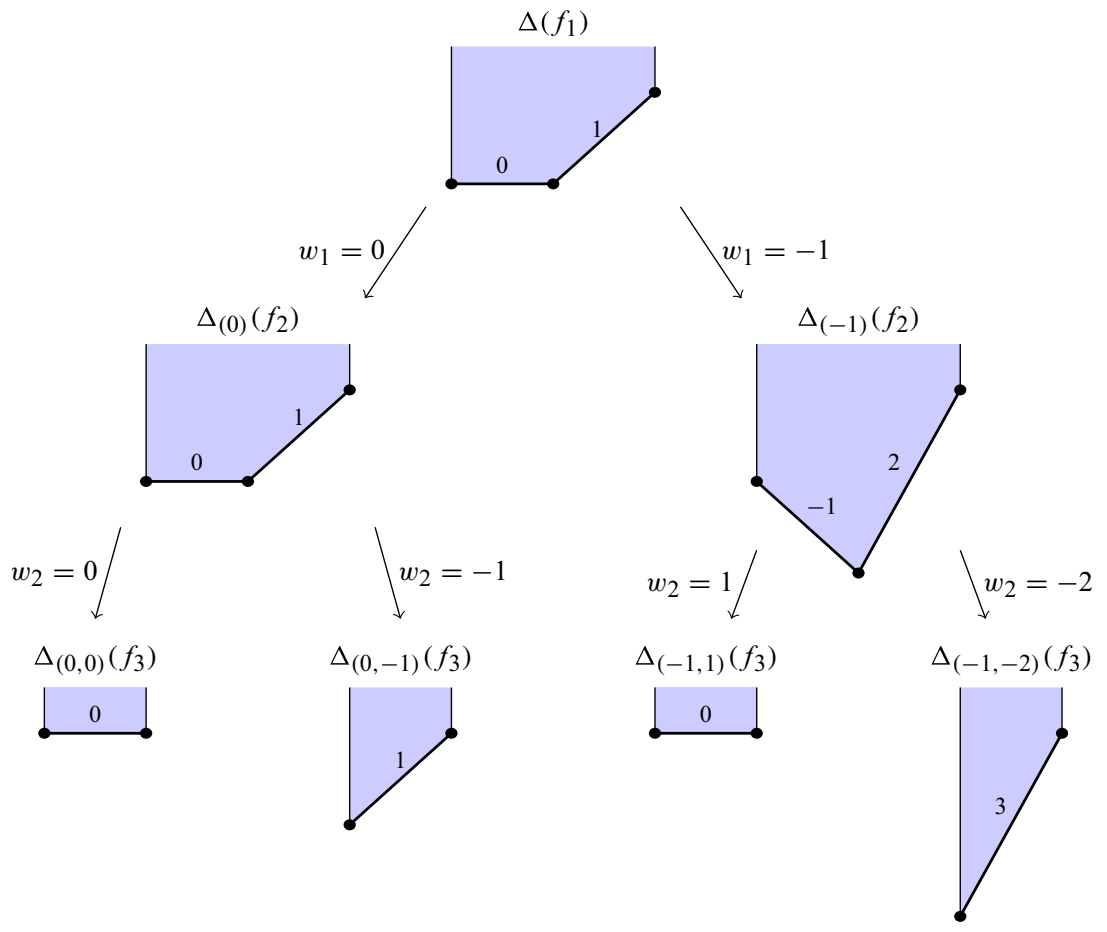

Fig. 2 A tree of unique Newton polygons

Example 2.13 (Computing entire tropical varieties) As mentioned in the beginning of the section, Algorithm 2.10 can be used to compute entire tropical varieties of zero-dimensional ideals. This is done by computing a triangular decomposition as in Proposition 2.4 and applying the algorithm to each triangular set, while exhausting all in Steps 4 and 6-7. For instance, consider the triangular set $F=\left\{f_{1}, f_{2}, f_{3}\right\} \subseteq$ $\mathbb{C}\{\{t\}\}\left[x_{1}, x_{2}, x_{3}\right]$ with

$$
f_{1}=t x_{1}^{2}+x_{1}+1, \quad f_{2}=t x_{2}^{2}+x_{1} x_{2}+1, \quad f_{3}=x_{3}+x_{1} x_{2} .
$$

Then $F$ admits several choices for slopes throughout the algorithm, and each choice in turn induces a new unique Newton polygon as illustrated in Fig. 2. Keeping track of all of them, allows us to reconstruct its entire tropical variety:

$$
\operatorname{Trop}(F)=\{(0,0,0),(0,-1,-1),(-1,1,0),(-1,-2,-3)\}
$$

We conclude this section by showing that any generic triangular set resembles Example 2.13 in the sense that its tropical variety is determined by a tree of unique Newton polygons.

Definition 2.14 We say a triangular set $F=\left\{f_{1}, \ldots, f_{n}\right\} \subseteq K[x]$ admits a tree of unique Newton polygons, if for all $k=1, \ldots, n$ and all weights $w=$ 
Fig. $3 \Delta_{w}(f)$ around a non-monomial vertex

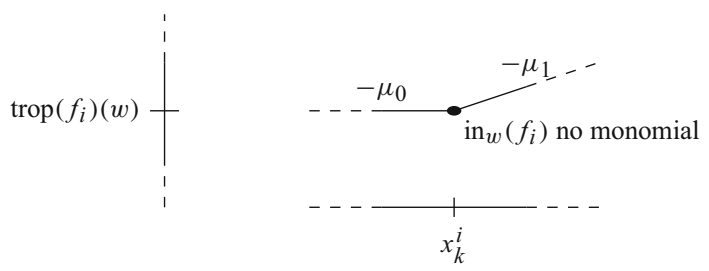

$\left(w_{1}, \ldots, w_{k-1}\right) \in \mathbb{R}^{k-1}$ with $w_{i} \in \Lambda_{\left(w_{1}, \ldots, w_{i-1}\right)}\left(f_{i}\right), i=1, \ldots, k-1$, the polynomial $f_{k}$ has a unique Newton polygon at $\left(w_{1}, \ldots, w_{k-1}\right)$.

Lemma 2.15 Consider $w \in \mathbb{R}^{k-1}$ and $f \in K\left[x_{1}, \ldots, x_{k}\right] \subseteq K\left[x_{1}, \ldots, x_{n}\right]$ such that $\left(\{w\} \times \mathbb{R}^{n-k+1}\right) \cap \operatorname{Trop}(f)$ has codimension $k$. Then $f$ has a unique Newton polygon at $w$ and

$$
\left(\{w\} \times \mathbb{R}^{n-k+1}\right) \cap \operatorname{Trop}(f)=\{w\} \times \bigcup_{\tilde{w} \in \Lambda_{w}(f)}\{\tilde{w}\} \times \mathbb{R}^{n-k}
$$

Proof Without loss of generality, assume that $k=n$. Suppose $f=\sum_{i=0}^{d} f_{i} \cdot x_{k}^{i}$ with $f_{i} \in K\left[x_{1}, \ldots, x_{k-1}\right]$ and assume that $f$ has no unique Newton polygon at $w$, i.e., that there exists a vertex $\left(i, \operatorname{trop}\left(f_{i}\right)(w)\right) \in \Delta_{w}(f)$ such that $\operatorname{in}_{w}\left(f_{i}\right)$ is no monomial. Let $\mu_{0}$ and $\mu_{1}$ be the negated slopes of the edges after and before the vertex respectively, see Fig. 3. Then, for any $w_{k} \in\left(\mu_{0}, \mu_{1}\right)$, we have $\operatorname{in}_{\left(w, w_{k}\right)}(f)=\operatorname{in}_{w}\left(f_{i}\right) \cdot x_{k}^{i}$, which is no monomial. This implies $\{w\} \times\left(\mu_{1}, \mu_{0}\right) \subseteq \operatorname{Trop}(f)$, contradicting the zerodimensionality of $\operatorname{Trop}(f)$.

Next, we show the equality. For the " $\supseteq$ " inclusion, let $\mu$ be a slope of an edge of $\Delta_{w}(f)$, say connecting the two vertices $v_{0}$ and $v_{1}$. Then, writing $e\left(v_{0}, v_{1}\right)$ for the edge connecting $v_{0}$ and $v_{1}$,

$$
\operatorname{in}_{(w, \mu)}(f)=\sum_{\left(i, \operatorname{trop}\left(f_{i}\right)(w)\right) \in e\left(v_{0}, v_{1}\right)} \operatorname{in}_{w}\left(f_{i}\right) \cdot x_{k}^{i} .
$$

For the converse inclusion, let $\left(w, w_{k}\right) \in \operatorname{Trop}(f)$. It is clear that for some bounded proper face $e \leq \Delta_{w}(f)$,

$$
\operatorname{in}_{\left(w, w_{k}\right)}(f)=\sum_{\left(i, \operatorname{trop}\left(f_{i}\right)(w)\right) \in e} \operatorname{in}_{w}\left(f_{i}\right) \cdot x_{k}^{i} .
$$

Note that $e$ cannot be zero-dimensional, as otherwise $\operatorname{in}_{\left(w, w_{k}\right)}(f)=\operatorname{in}_{\left(w, w_{k}^{\prime}\right)}(f)$ for all $w_{k}^{\prime} \in \mathbb{R}$, contradicting the zero-dimensionality of $\operatorname{Trop}(f)$. Hence, $e$ has to be an edge and, consequently, $w_{k}$ is the slope of $e$.

Proposition 2.16 For a triangular set $F=\left\{f_{1}, \ldots, f_{n}\right\} \subseteq K[x]$ the following are equivalent:

1. $\operatorname{dim} \bigcap_{i=1}^{k} \operatorname{Trop}\left(f_{i}\right)=n-k$ for all $k=1, \ldots, n$,

2. $F$ is a tropical basis.

Moreover, if $F$ is a tropical basis, then it admits a tree of unique Newton polygons. 
Proof We first show that (1) implies that $F$ is a tropical basis and that it admits a tree of unique Newton polygons. By definition, we have $\operatorname{Trop}\left(f_{1}\right)=\bigcup_{w_{1} \in \Lambda\left(f_{1}\right)}\left\{w_{1}\right\} \times \mathbb{R}^{n-1}$. Applying Lemma 2.15 repeatedly, we see that for $w_{1} \in \Lambda\left(f_{1}\right)$, the polynomial $f_{2}$ has a unique Newton polygon at $w_{1}$ with

$$
\left(\left\{w_{1}\right\} \times \mathbb{R}^{n-1}\right) \cap \operatorname{Trop}\left(f_{2}\right)=\left\{w_{1}\right\} \times \bigcup_{w_{2} \in \Lambda_{w_{1}}\left(f_{2}\right)}\left\{w_{2}\right\} \times \mathbb{R}^{n-2},
$$

and, for $w_{1} \in \Lambda\left(f_{1}\right)$ and $w_{2} \in \Lambda_{w_{1}}\left(f_{2}\right), f_{3}$ has a unique Newton polygon at $\left(w_{1}, w_{2}\right)$ with

$$
\left(\left\{\left(w_{1}, w_{2}\right)\right\} \times \mathbb{R}^{n-2}\right) \cap \operatorname{Trop}\left(f_{3}\right)=\left\{\left(w_{1}, w_{2}\right)\right\} \times \underset{w_{3} \in \Lambda_{\left(w_{1}, w_{2}\right)}\left(f_{3}\right)}{\bigcup}\left\{w_{3}\right\} \times \mathbb{R}^{n-3},
$$

and so forth. This shows on the one hand that $F$ admits a tree of unique Newton polygons and on the other hand that any point in $\bigcap_{i=1}^{n} \operatorname{Trop}\left(f_{i}\right)$ corresponds to the component-wise valuation of a point in $V(F)$, implying that $F$ is a tropical basis.

It remains to show that if (1) is not true, then $F$ is no tropical basis. Assume for the sake of simplicity that $\operatorname{dim} \operatorname{Trop}\left(f_{1}\right) \cap \operatorname{Trop}\left(f_{2}\right)=n-1$. Because $\operatorname{Trop}\left(f_{1}\right)=$ $\bigcup_{w_{1} \in \Lambda\left(f_{1}\right)}\left\{w_{1}\right\} \times \mathbb{R}^{n-1}$ and $\operatorname{Trop}\left(f_{2}\right)$ is invariant under translation by $\{(0,0)\} \times \mathbb{R}^{n-2}$, there necessarily exist

$$
\{\lambda\} \times\left[\mu_{1}, \mu_{2}\right] \times \mathbb{R}^{n-2} \subseteq \operatorname{Trop}\left(f_{1}\right) \cap \operatorname{Trop}\left(f_{2}\right),
$$

for $\lambda \in \Lambda\left(f_{1}\right)$ and a nontrivial $\left[\mu_{1}, \mu_{2}\right] \subseteq \mathbb{R}$. Consequently,

$$
\{\lambda\} \times\left[\mu_{1}, \mu_{2}\right] \times\{(0, \ldots, 0)\} \subseteq \bigcap_{i=1}^{n} \operatorname{Trop}\left(f_{i}\right),
$$

and since $\bigcap_{i=1}^{n} \operatorname{Trop}\left(f_{i}\right)$ is not zero-dimensional, $F$ cannot be a tropical basis of the zero-dimensional ideal it generates.

From Proposition 2.16, we conclude that a generic triangular set is a tropical basis and admits a tree of unique Newton polygons in the following sense:

Corollary 2.17 Let $\left(K^{*}\right)^{N} \subseteq K[x]^{n}$ be the coefficient space of all triangular sets with fixed support. Then, in the topology induced by the valuation, there exists an open dense set $\mathcal{U} \subseteq\left(K^{*}\right)^{N}$ such that any triangular set $F \in \mathcal{U}$ is a tropical basis and admits a tree of unique Newton polygons.

Proof Consider the component-wise valuation $v:\left(K^{*}\right)^{N} \rightarrow \mathbb{R}^{N}$. There exists an Euclidean open dense subset $U \subseteq \mathbb{R}^{n}$ such that the tropical hypersurfaces of any triangular set $F \in\left(K^{*}\right)^{N}$ with $v(F) \in U$ intersect transversally as in Proposition 2.16 (1). As $v$ is continuous, its preimage $\mathcal{U}:=v^{-1} U \subseteq\left(K^{*}\right)^{N}$ is also open and dense. 


\section{Computing tropical starting points}

In this section, we use Algorithm 2.10 to compute points on higher-dimensional tropical varieties. This is done by reducing the dimension to zero by intersecting with randomly chosen hyperplanes. Moreover, we will use the algorithm to sample random maximal Gröbner cones on the tropical Grassmannians $\mathcal{G}_{3,7}, \mathcal{G}_{4,7}, \mathcal{G}_{3,8}, \mathcal{G}_{4,8}$ and show that the latter two are not simplicial.

Proposition 3.1 Let $I \unlhd K[x]$ be a prime ideal of dimension d and $X=V(I)$ its corresponding irreducible affine variety such that $X \cap\left(K^{*}\right)^{n} \neq \emptyset$. W.l.o.g. let $\left\{x_{1}, \ldots, x_{d}\right\}$ be algebraically independent modulo I. Then there exists a non-empty, Zariski open subset $U \subseteq\left(K^{*}\right)^{d}$ such that for all $\lambda \in U$

$$
\emptyset \neq X \cap V\left(\left\langle x_{i}-\lambda_{i} \mid i=1, \ldots, d\right\rangle\right) \subseteq\left(K^{*}\right)^{n}
$$

and $\operatorname{dim}\left(X \cap V\left(\left\langle x_{i}-\lambda_{i} \mid i=1, \ldots, d\right\rangle\right)\right)=0$.

Proof Abbreviating $H_{\lambda}:=V\left(\left\langle x_{i}-\lambda_{i} \mid i=1, \ldots, d\right\rangle\right)$, it is clear that there exists a Zariski open $U_{0} \subseteq\left(K^{*}\right)^{d}$ with $\emptyset \neq X \cap H_{\lambda}$ and $\operatorname{dim}\left(X \cap H_{\lambda}\right)=0$. Now consider the set in which the inclusion does not hold. It naturally decomposes into $n-d$ subsets:

$$
A:=\left\{\lambda \in\left(K^{*}\right)^{d} \mid X \cap H_{\lambda} \nsubseteq\left(K^{*}\right)^{n}\right\}=\bigcup_{i=d+1}^{n} \underbrace{\left\{\lambda \in\left(K^{*}\right)^{d} \mid \exists z \in X \cap H_{\lambda}: z_{i}=0\right\}}_{=: A_{i}} .
$$

As $U$ can be chosen to be $U_{0} \backslash \bar{A}$, where $\overline{(\cdot)}$ denotes the Zariski closure in $\left(K^{*}\right)^{d}$, it suffices to show that $\bar{A}_{i} \neq\left(K^{*}\right)^{d}$. This is easy to see: Because $X$ is irreducible and $X \cap\left(K^{*}\right)^{n} \neq \emptyset$, we necessarily have $\operatorname{dim}\left(X \cap V\left(x_{i}\right)\right)<d$ for all $i=d+1, \ldots, n$. In particular, $\operatorname{dim} \pi\left(X \cap V\left(x_{i}\right)\right)<d$, where $\pi: K^{n} \rightarrow K^{d}$ is the canonical projection onto the first $d$ coordinates. And, by construction, $A_{i} \subseteq \pi\left(X \cap V\left(x_{i}\right)\right)$.

Proposition 3.1 can be reformulated into the following algorithm.

\section{Algorithm 3.2 (Tropical point)}

Input: $I \unlhd K[x]$ prime ideal with $V(I) \cap\left(K^{*}\right)^{n} \neq \emptyset$.

Output: $w \in \operatorname{Trop}(I)$.

1: Compute a maximal algebraically indep. set modulo $I$, say $\left\{x_{1}, \ldots, x_{d}\right\}$.

2: repeat

3: Pick $z=\left(z_{1}, \ldots, z_{d}\right) \in\left(K^{*}\right)^{d}$ randomly.

4: Set $I_{z}$ to be the image of $I$ under the substitution map

$$
K\left[x_{1}, \ldots, x_{n}\right] \rightarrow K\left[x_{d+1}, \ldots, x_{n}\right], \quad x_{i} \mapsto \begin{cases}z_{i} & \text { if } i \leq d \\ x_{i} & \text { else }\end{cases}
$$

5: until $\operatorname{dim}\left(I_{z}\right)=0$ and $V\left(I_{z}\right) \subseteq\left(K^{*}\right)^{n-d}$

6: Compute a triangular set $F \subseteq K\left[x_{d+1}, \ldots, x_{n}\right]$ with $\sqrt{I_{z}} \subseteq\langle F\rangle$.

7: Compute a point $\left(w_{d+1}, \ldots, w_{n}\right) \in \operatorname{Trop}(F)$ using Algorithm 2.10.

8: return $\left(v(z), w_{d+1}, \ldots, w_{n}\right)$ 
Remark 3.3 1. Randomized algorithms such as Algorithm 3.2 are commonly referred to as Las Vegas algorithms. This means that its result is always correct, however it only has an expected finite runtime. Nevertheless, Proposition 3.1 shows that generic choices of $z$ in Step 3 will lead to termination.

2. Note that the set of all $w \in \mathbb{R}^{d}$ such that $\{w\} \times \mathbb{R}^{n-d}$ does not intersect any lowerdimensional Gröbner polyhedra on $\operatorname{Trop}(I)$ is open and dense in the Euclidean topology. Hence generic choices of $z \in\left(K^{*}\right)^{d}$ in Step 3 will also guarantee that the resulting tropical point will lie in the relative interior of a maximal Gröbner polyhedra on the tropical variety.

3. It is possible to eliminate the randomness by computing stable intersections with affine hyperplanes, as in a recent work of Jensen and $\mathrm{Yu}$ [18]. However, this requires one transcendental extension of $K$ per hyperplane, which is not feasible in high codimension.

We will briefly define the examples of our interest.

Definition 3.4 Let $k, n \in \mathbb{N}_{>0}$ with $1 \leq k \leq n$. The tropical Grassmannian $\mathcal{G}_{k, n} \subseteq$ $\mathbb{R}^{\left(\begin{array}{l}n \\ k\end{array}\right)}$ is defined to be the tropicalization of the ideal $\operatorname{Grass}(k, n) \unlhd K[p]$, where the variables of the ring $K[p]:=K\left[p_{i_{1} \cdots i_{k}} \mid 1 \leq i_{1}<\ldots<i_{k} \leq n\right]$ represent the $k \times k$ minors of any $k \times n$ matrix and the ideal $\operatorname{Grass}(k, n)$ is generated by all Plücker relations amongst them, see [21, Sect. 4.3]. We consider the variables of $K[p]$ to be sorted lexicographically, i.e.,

$$
p_{i_{1} \cdots i_{k}}>p_{j_{1} \cdots j_{k}}: \Longleftrightarrow \quad \exists 1 \leq l<k: i_{1}=j_{1}, \ldots, i_{l-1}=j_{l-1} \text { and } i_{l}>j_{l} \text {. }
$$

Moreover, we define the ideal $\operatorname{Det}(k, n) \unlhd K\left[x_{11}, x_{12}, \ldots, x_{n n}\right]$ to be the ideal generated by the $k \times k$ minors of the matrix $\left(x_{i j}\right)_{i, j=1, \ldots, n}$.

Example $3.5\left(\mathcal{G}_{2,5}\right)$ Let $K=\mathbb{C}\{\{t\}\}$. We demonstrate Algorithm 3.2 on the tropical Grassmannian $\mathcal{G}_{2,5}$. Its ideal is given by

$$
\begin{aligned}
\operatorname{Grass}(2,5)= & \left\langle p_{34} p_{25}-p_{24} p_{35}+p_{23} p_{45}, p_{34} p_{15}-p_{14} p_{35}+p_{13} p_{45},\right. \\
& p_{24} p_{15}-p_{14} p_{25}+p_{12} p_{45}, p_{23} p_{15}-p_{13} p_{25}+p_{12} p_{35}, \\
& \left.p_{23} p_{14}-p_{13} p_{24}+p_{12} p_{34}\right\rangle \unlhd K\left[p_{12}, p_{13}, \ldots, p_{45}\right] .
\end{aligned}
$$

It is 7-dimensional with maximal independent set $\left\{p_{15}, p_{23}, p_{24}, \ldots, p_{45}\right\}$.

Choosing $\left(z_{15}, z_{23}, z_{24}, \ldots, z_{45}\right):=(t, \ldots, t)$ yields $I_{z}=\left\langle p_{12}, p_{13}, p_{14}\right\rangle$, which means that the choice is not generic in the sense of Proposition 3.1. Choosing $\left(t, t^{5}, t^{3}, t^{7}, t^{8}, t^{2}, t^{9}\right)$ on the other hand yields $I_{z}$ generated by the triangular set 


$$
p_{12}+\left(t^{3}-1\right), \quad t^{6} \cdot p_{13}+\left(t^{7}-1\right), \quad t^{2} \cdot p_{14}+\left(t^{4}-1\right) .
$$

Looking at the Newton polygons, we conclude that $w:=(0,-6,-2) \in \operatorname{Trop}\left(I_{z}\right)$. Thus $(v(z), w)=(0,-6,-2,1,5,3,7,8,2,9) \in \operatorname{Trop}(I)$.

In addition to computing starting points for the tropical traversals, Algorithm 3.2 can be used to sample random points on tropical varieties.

Example $3.6\left(\mathcal{G}_{k, n}\right.$ for $k \in\{3,4\}$ and $\left.n \in\{7,8\}\right)$ Using Algorithm 3.2, we sampled random maximal cones on higher tropical Grassmannians ignoring symmetry. This was done by computing Gröbner cones around random tropical points, dismissing those of lower dimension and duplicates. We analyzed over 1000 distinct maximal cones on each of $\mathcal{G}_{3,7}, \mathcal{G}_{4,7}$ and $\mathcal{G}_{3,8}$, as well as over 100 distinct maximal cones on the tropical variety of $\mathcal{G}_{4,8}$.

All cones were invariant under tensoring with $\mathbb{F}_{2}$, which is not surprising for $\mathcal{G}_{3,7}$ : Even though Speyer and Sturmfels showed that $\mathcal{G}_{3,7}$ depends on the characteristic of the ground field, in fact it is the smallest tropical Grassmannian depicting this behavior [25, Thm. 3.7], Herrmann, Jensen, Joswig and Sturmfels showed that, out of the 252000 maximal cones of $\mathcal{G}_{3,7}$, this is only visible on a single cone, the Fano cone [12, Thm. 2.1].

Of the 1000 Gröbner cones sampled from each of $\mathcal{G}_{3,7}$ and $\mathcal{G}_{4,7}$, every single one was simplicial, which was expected as $\mathcal{G}_{3,7}$ is known to be simplicial [12, Thm. 2.1] and $\mathcal{G}_{4,7}=\mathcal{G}_{3,7}$ by duality. In the 1000 and 100 Gröbner cones sampled from $\mathcal{G}_{3,8}$ and $\mathcal{G}_{4,8}$ respectively, each contained exactly one cone which was not simplicial, see the proof of Theorem 3.7.

Not much is known on $\mathcal{G}_{3,8}$ and $\mathcal{G}_{4,8}$, but there is a complete description of the Dressian $\mathcal{D}_{3,8}$ by Herrmann, Joswig and Speyer [13, Thm. 31], which is a natural tropical prevariety containing $\mathcal{G}_{3,8}$ that parametrizes all tropical linear spaces. It is known that all rays of $\mathcal{D}_{3,7}$ and $\mathcal{D}_{3,8}$ are also rays of $\mathcal{G}_{3,7}$ and $\mathcal{G}_{3,8}$ respectively, and that $\mathcal{G}_{3,7}$ contains rays which are not rays of $\mathcal{D}_{3,7}$. Our sampling also revealed that this holds for $\mathcal{G}_{3,8}$. In fact, none of the 126 tested rays of $\mathcal{G}_{3,8}$ were rays of $\mathcal{D}_{3,8}$, a concrete example is the ray generated by the following vector:

$$
\begin{aligned}
& (0,-1,1,1,-1,0,0,0,0,1,0,1,1,1,1,1,0,0,1,1,-2,0,0,-1,-1,0,-1,-1, \\
& 0,-1,0,0,0,0,0,0,1,1,1,1,1,0,0,1,1,0,0,1,1,0,0,0,0,0,0,0) \in \mathbb{R}^{\left(\begin{array}{l}
8 \\
3
\end{array}\right)} .
\end{aligned}
$$

This is somewhat in stark contrast to $\mathcal{G}_{3,7}$ and $\mathcal{D}_{3,7}$, as out of the 721 rays of the Grassmannian 616 were rays of the Dressian [12, Thm. 2.2].

As an immediate result, we obtain:

Theorem 3.7 The tropical Grassmannian $\mathcal{G}_{d, n}$ is not simplicialfor $d=3,4$ and $n=8$. 
Proof Consider the following two points which lie on $\mathcal{G}_{3,8}$ and $\mathcal{G}_{4,8}$ respectively:

$$
\begin{aligned}
w_{3,8}= & (2,10,7,10,2,2,2,10,7,10,9,6,9,12, \\
& 12,12,9,6,9,12,5,2,5,5,2,5,5,2,5,1, \\
& 7,7,7,7,7,9,6,9,12,12,12,9,6,9,12,9, \\
& 7,9,12,7,7,7,7,7,7,7) \in \mathbb{R}^{\left(\begin{array}{l}
8 \\
3
\end{array}\right)}, \\
w_{4,8}= & (2,1,8,1,8,14,21,15,21,11,14,11,21, \\
& 16,21,5,12,6,12,4,5,4,12,7,12,17,12, \\
& 17,19,19,19,17,12,17,19,14,21,15,21, \\
& 11,14,11,21,16,21,21,15,21,21,19, \\
& 21,21,16,21,19,17,12,17,19,19,19,17,12, \\
& 17,19,19,19,19,19,19) \in \mathbb{R}^{\left(\begin{array}{l}
8 \\
4
\end{array}\right) .}
\end{aligned}
$$

A corresponding reduced Gröbner basis of Grass $(3,8)$ under the weighted monomial ordering with weight vector $w_{3,8}$ and lexicographical tiebreaker has 686 elements of degrees ranging from 2 to 6 , while the reduced Gröbner basis for $\operatorname{Grass}(4,8)$ has 1157 elements of degrees ranging from 2 to 8 .

The Gröbner cone containing $w_{3,8}$ in its relative interior is of dimension 16 , generated by 9 rays and a lineality space of dimension 8 , and the Gröbner cone with $w_{4,8}$ in its relative interior is of dimension 17 , generated by 10 rays and a lineality space of dimension 8 . Hence both cones are maximal-dimensional in their respective tropical varieties and not simplicial.

We conclude the section with some timings.

Timings 3.8 Figure 4 compares three different algorithms for computing points on tropical varieties:

GFAN 0.6.2: an experimental algorithm based on Chan's work on tropical curves [3, Chap. 4], and Jensen and Yu's work on stable intersections [18].

GFAN 0.5: [2, Algorithm 9], a random traversal of the Gröbner fan while testing all rays for containment in the tropical variety. It can also be found in SINGULAR, however that implementation is slower than GFAN.

SINGULAR 4.1.0: Algorithm 3.2, as implemented in tropicalNewton. 1 ib. As GFAN additionally computes a corresponding reduced Gröbner basis, we also provide analogous timings in SINGULAR.

We would like to stress that these timings merely serve as a comparison of the algorithms and not as a showcase of the computational reach of the two systems involved. For instance, points on tropical Grassmannians can also be computed via the tropical Stiefel map, see [13, Prop. 12] and [8]. In fact, $\mathcal{G}_{3,7}$ has been previously computed

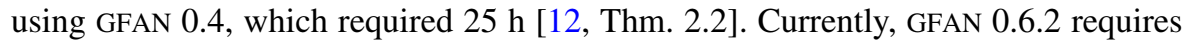
65 min, while SINGULAR 4.1.0 requires 10 min.

All computations were run on a machine with Intel E5-2643v3 (3.4 GHz) processors running Gentoo 4.4.6 and were aborted after exceeding 7 CPU days. See Definition 3.4 


\begin{tabular}{|l|rrrr|}
\hline & GFAN 0.6 .2 & GFAN 0.5 & \multicolumn{3}{c|}{ SINGULAR 4.1.0 } \\
Det(2,5) & & & $w \in \operatorname{Trop}(I)$ & GB under $>w$ \\
$\operatorname{Det}(3,5)$ & 1 & 1 & 1 & 1 \\
$\operatorname{Det}(2,6)$ & 140 & 7 & 1 & 1 \\
$\operatorname{Det}(3,6)$ & 5 & 1 & 1 & 1 \\
$\operatorname{Det}(4,6)$ & 1800 & 900 & 8 & 1 \\
$\operatorname{Det}(5,6)$ & - & 1100 & 41 & 1 \\
$\operatorname{Grass}(3,7)$ & - & 100 & 7 & 1 \\
$\operatorname{Grass}(3,8)$ & 140 & - & 1 & 1 \\
$\operatorname{Grass}(3,9)$ & - & - & 3 & 1 \\
$\operatorname{Grass}(4,7)$ & - & - & 19 & 12 \\
$\operatorname{Grass}(4,8)$ & 170 & - & 1 & 3 \\
$\operatorname{Grass}(4,9)$ & - & - & 9 & 900 \\
$\operatorname{Grass}(5,8)$ & - & - & 230 & 1 \\
\hline
\end{tabular}

Fig. 4 Timings in seconds, '-' were aborted after 7 CPU days

for the definitions of $\operatorname{Det}(k, n)$ and $\operatorname{Grass}(k, n)$. All examples are considered over $\mathbb{C}\{\{t\}\}$.

Timings 3.9 Consider the computation of tropical points for the family of onedimensional ideals generated by $F=\left\{f_{1}, \ldots, f_{n}\right\} \subseteq \mathbb{Q}\left[x_{0}, x_{1}, \ldots, x_{n}\right] \subseteq$ $\mathbb{C}\{\{t\}\}\left[x_{0}, x_{1}, \ldots, x_{n}\right]$ given by

$$
f_{k}=x_{k}^{2}-q_{k} x_{0}\left(\sum_{i=1}^{k-1} x_{i}\right)+q_{k} x_{0}^{2}, \quad \text { where } q_{k} \in \mathbb{N} \text { is the } k \text {-th prime. }
$$

While substituting $x_{0} \mapsto t$ directly yields triangular sets, we described in Example 2.12 how our Algorithm 2.10 struggles with them: It requires a degree $2^{n}$ field extension of $\mathbb{Q}$, which results in a runtime of $8 \mathrm{~s}$ for $n=13$, roughly doubling with each increase of $n$.

However, for [2, Algorithm 9], this family is completely trivial: As $F$ is already a reduced Gröbner basis for a suitable ordering, it is easy to verify that its ideal is one-dimensional and has a one-dimensional homogeneity space generated by $(1,1, \ldots, 1) \in \mathbb{R}^{n+1}$. Hence [2, Algorithm 9] immediately obtains its tropical variety, which is equal to its homogeneity space. This shows in the runtime of both GFAN 0.5 and GFAN 0.6.2, which terminate instantaneously for $n=13$ and whose runtimes remain under 1 second for $n<120$.

\section{Computing tropical links}

In this section, we use Algorithm 2.10 to compute links of a tropical variety around its one-codimensional Gröbner polyhedra. This is done in two steps. First we intersect the link with a subspace to reduce it to a one-dimensional polyhedral fan. Afterwards, we intersect the fan with affine hypersurfaces to determine all its rays. 
Fig. 5 A tropical link of a tropical cubic curve

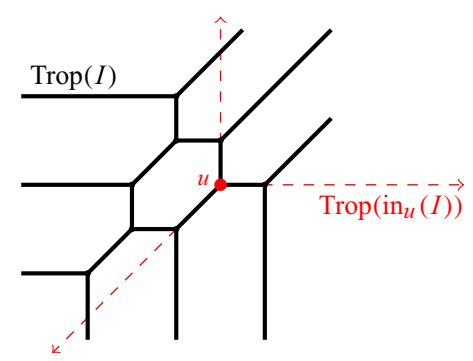

Definition 4.1 We refer to $\operatorname{Trop}(I)$ as a tropical link, if it is a polyhedral fan and has a one-codimensional lineality space.

Remark 4.2 Let $u \in \operatorname{Trop}(I)$ sit in the relative interior of a one-codimensional Gröbner polyhedron. Then $\operatorname{Trop}\left(\mathrm{in}_{u}(I) \otimes_{\mathfrak{K}} \mathfrak{K}\{\{t\}\}\right)$ is a tropical link which describes $\operatorname{Trop}(I)$ locally around $u$, see Fig. 5. Its lineality space is the linear subspace spanned by the Gröbner polyhedra after moving $u$ to the origin.

The reduction to dimension zero relies on the following result on the intersection of tropical varieties by Osserman and Payne. From it, we can immediately write down our algorithm.

Theorem 4.3 ([24, Thm. 1.1]) Let $X$ and $X^{\prime}$ be two affine subvarieties. If $\operatorname{Trop}(X) \cap$ $\operatorname{Trop}\left(X^{\prime}\right)$ has codimension codim $\operatorname{Trop}(X)+\operatorname{codim} \operatorname{Trop}\left(X^{\prime}\right)$ in a neighborhood of $w$, then $w$ is contained in $\operatorname{Trop}\left(X \cap X^{\prime}\right)$.

Corollary 4.4 Let $\operatorname{Trop}(I)$ be a $(d+1)$-dimensional tropical link and $H$ its $d$ dimensional lineality space. Suppose $H \cap \operatorname{Lin}\left(e_{d+1}, \ldots, e_{n}\right)=\{0\}$. Then for any $z \in\left(K^{*}\right)^{d}$ we have

$$
\operatorname{Trop}(I) \cap\left(\{v(z)\} \times \mathbb{R}^{n-d}\right)=\operatorname{Trop}\left(I+\left\langle x_{i}-z_{i} \mid i=1, \ldots, d\right\rangle\right),
$$

and $\operatorname{Trop}\left(I+\left\langle x_{i}-z_{i} \mid i=1, \ldots, d\right\rangle\right)$ is a one-dimensional tropical link with lineality space $\{0\}$.

Corollary 4.5 Let $\operatorname{Trop}(I)$ be a one-dimensional tropical link with lineality space $\{0\}$. Then for any $z \in K^{*}$ with $v(z) \neq 0$ we have

$$
\operatorname{Trop}(I) \cap\left(\{v(z)\} \times \mathbb{R}^{n-1}\right)=\operatorname{Trop}\left(I+\left\langle x_{1}-z\right\rangle\right),
$$

and $\operatorname{Trop}\left(I+\left\langle x_{1}-z\right\rangle\right)$ is either empty or zero-dimensional.

\section{Algorithm 4.6 (Tropical link)}

Input: $I \unlhd K[x]$ such that $\operatorname{Trop}(I)$ is a $(d+1)$-dimensional tropical link with $d$ dimensional lineality space $H$.

Output: $W \subseteq \mathbb{R}^{n}$ such that $\operatorname{Trop}(I)=\bigcup_{w \in W} w \cdot \mathbb{R}_{\geq 0}+H$.

1: Find $A \subseteq\{1, \ldots, n\}$ such that $|A|=n-d$ and $H \cap \operatorname{Lin}\left(e_{i} \mid i \in A\right)=\{0\}$, say $A=\{d+1, \ldots, n\}$. 
2: Let $J$ be the image of $I$ under the substitution map

$$
K\left[x_{1}, \ldots, x_{n}\right] \rightarrow K\left[x_{d}, \ldots, x_{n}\right], \quad x_{i} \mapsto \begin{cases}1 & \text { if } i<d, \\ x_{i} & \text { else }\end{cases}
$$

3: for $i=d, \ldots, n$ do

4: $\quad$ Let $J_{i}^{ \pm}$be images of $J$ under the maps $x_{i} \mapsto p^{ \pm 1}$ respectively.

5: if $J_{i}^{ \pm} \neq\langle 1\rangle$ then

6: $\quad$ Compute $T_{i}^{ \pm}=\operatorname{Trop}\left(J_{i}^{ \pm}\right)$using Algorithm 2.10.

7: $\quad$ Set

$$
\begin{array}{r}
W_{i}^{ \pm}:=\left\{\left(0, \ldots, 0, w_{d}, \ldots, w_{i-1}, \pm 1, w_{i+1}, \ldots, w_{n}\right) \in \mathbb{R}^{n} \mid\right. \\
\left.\left(w_{d}, \ldots, w_{i-1}, w_{i+1}, \ldots, w_{n}\right) \in T_{i}^{ \pm}\right\} .
\end{array}
$$

8: $\quad$ Scale elements of $W_{i}^{ \pm}$positively so that they are primitive in $\mathbb{Z}^{n}$.

9: return $W:=\bigcup_{i=d}^{n} W_{i}^{ \pm}$

Remark 4.7 (Comparison with existing algorithms) The idea of computing tropical links by reducing the dimension is not new. Andrew Chan has designed an algorithm which computes tropical links via projection and reconstruction [3, Sect. 4], based on existing techniques developed by Hept and Theobald [11].

In both algorithms, the polyhedral computations are timewise irrelevant compared to the polynomial computations, which contain three potential bottlenecks (assuming $K=\mathbb{C}\{\{t\}$ and the use of [9, Algorithm 4.7.8] for the triangular decomposition necessary before applying Algorithm 2.10 to $J_{i}^{ \pm}$):

\section{[3, Algorithm 4.2.5, Step 1]}

Computing elimination ideals

[3, Algorithm 4.2.14, Step 6]

computing initial ideals

[3, Algorithm 4.2.14, Step 6]

Computing saturations
(Algorithm 4.6, Step 6)

Computing lexicographical Gröbner bases (Algorithm 4.6, Step 6)

Computing triangular decompositions

(Algorithm 2.10, Step 6)

Computing Newton-Puiseux expansions

Experiments suggest that, in both algorithms, the latter two bottlenecks are timewise insignificant compared to the first. In fact, for Algorithm 4.6, constructing the triangular decomposition from a lexicographical Gröbner basis is polynomial [20, Sect. 7], as is the construction of the Newton-Puiseux expansion [5]. Hence, the main bottleneck in both algorithms lies in the computation of Gröbner bases with respect to elimination orderings.

However, the key difference is that these Gröbner basis computations in [3, Algorithm 4.2.5] involve the one-dimensional input ideal $I$, whereas the ideals $J_{i}^{ \pm}$in Algorithm 4.6 are all zero-dimensional. For these ideals we not only have better complexity bounds [19], but also techniques such as fglm [7], which speed up our calculations drastically. For instance, in the following Example 4.8 and in SINGULAR 4.1.0, a lexicographical Gröbner basis of $J_{i}^{ \pm}$required only 30 seconds of computation while an elimination ideal of $I$ required $25 \mathrm{~min}$. 


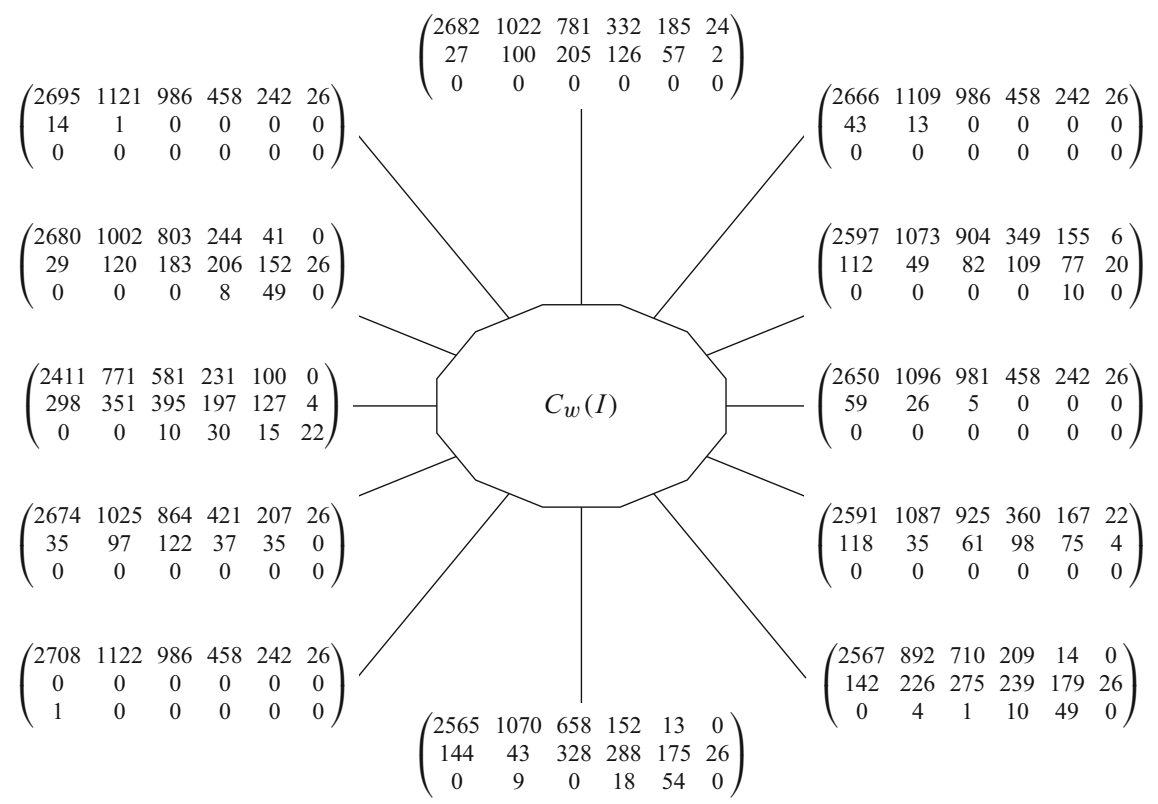

Fig. 6 Reduced Gröbner bases around a maximal cone in $\mathcal{G}_{4,9}$

Example 4.8 $\left(\mathcal{G}_{4,9}\right)$ Let $K=\mathbb{C}\{\{t\}\}$ and $I=\operatorname{Grass}(4,9)$. Its tropical variety $\mathcal{G}_{4,9} \subseteq$ $\mathbb{R}^{126}$ is of dimension 21 with a homogeneity space of dimension 9. Using Algorithm 2.10, one possible tropical point that lies in the interior of a maximal cone is

$$
\begin{aligned}
& w:=(1,1,3,4,4,7,8,9,9,9,10,10,9,5,10,10,10, \\
& 4,4,12,13,13,13,13,5,17,18,18,18,18, \\
& 9,19,19,19,19,8,9,10,10,10,11,11,10,7,11,11,12,12, \\
& 12,12,11,13,13,13,13,18, \\
& 19,19,19,19,10,20,20,20,20,20,20,20,20,20,10,10,10, \\
& 10,12,13,13,13,13,11, \\
& 17,18,18,18,18,11,19,19,19,19,18,19,19,19,19,10,20, \\
& 20,20,20,20,20,20,20, \\
& 20,18,19,19,19,19,11,20,20,20,20,20,20,20,20,20,20, \\
& 20,20,20,20,20) \in \mathbb{R}_{\left(\begin{array}{c}
9 \\
4
\end{array}\right) .}
\end{aligned}
$$

The reduced Gröbner basis of the initial ideal under $w$ with respect to the reverse lexicographical ordering consists of 5543 binomials with degrees ranging from 2 to 7. The Gröbner cone $C_{w}(I)$ is simplicial with its 12 facets. Figure 6 shows some data on the reduced Gröbner bases of the saturated initial ideals under weight vectors 
on the facets of $C_{w}(I)$. The rows represent binomials, trinomials and quadrinomials respectively and the columns represent degrees 2 to 7, i.e., the entry in row $i$ and column $j$ is the number of Gröbner basis elements with $i+1$ monomials and of degree $j+1$.

The computation of the 12 tropical links using Algorithm 4.6 took 7 min, while all attempts to compute any of the 12 tropical prevarieties failed to terminate within an hour, even using the newly developed techniques by Jensen et al. [17]. Similarly, computing any of the elimination ideals necessary in [3, Algorithm 4.2.5] required 25 min in SINGULAR. All tropical links are 3-valent, i.e., each facet is adjacent to exactly three maximal cones in the tropical variety.

Acknowledgements Open access funding provided by the Max Planck Society. The authors would like to thank Michael Joswig and Benjamin Schröter for their feedback on a previous version of the article.

Open Access This article is distributed under the terms of the Creative Commons Attribution 4.0 International License (http://creativecommons.org/licenses/by/4.0/), which permits unrestricted use, distribution, and reproduction in any medium, provided you give appropriate credit to the original author(s) and the source, provide a link to the Creative Commons license, and indicate if changes were made.

\section{References}

1. Allamigeon, X., Benchimol, P., Gaubert, S., Joswig, M.: Combinatorial simplex algorithms can solve mean payoff games. SIAM J. Optim. 24(4), 2096-2117 (2014)

2. Bogart, T., Jensen, A.N., Speyer, D., Sturmfels, B., Thomas, R.R.: Computing tropical varieties. J. Symb. Comput. 42(1-2), 54-73 (2007). https://doi.org/10.1016/j.jsc.2006.02.004

3. Chan, A.: Gröbner Bases Over Fields with Valuation and Tropical Curves by Coordinate Projections. $\mathrm{PhD}$ thesis. University of Warwick, (2013)

4. Chan, A.J, Maclagan, D.: Groebner bases over fields with valuations. Math. Comput. (2013). eprint: arXiv:1303.0729

5. Chistov, A.L.: Polynomial complexity of the Newton-Puiseux algorithm. In: Grushka, J., et al. (eds.) Mathematical Foundations of Computer Science 1986. Lecture Notes in Computer Science, vol. 233, pp. 247-255. Springer, Berlin (1986). https://doi.org/10.1007/Fb0016248

6. Decker, W., Greuel, G.-M., Pfister, G., Schnemann, H.: Singular 4-1-0-A computer algebra system for polynomial computations. http://www.singular.uni-kl.de. (2016)

7. Faugére, J.C., Gianni, P., Lazard, D., Mora, T.: Efficient computation of zerodimensional Gröbner bases by change of ordering. J. Symb. Comput. 16(4), 329-344 (1993). https://doi.org/10.1006/jsco. 1993.1051

8. Fink, A., Rincón, F.: Stiefel tropical linear spaces. J. Combin. Theory Ser. A 135, 291-331 (2015). https://doi.org/10.1016/j.jcta.2015.06.001

9. Greuel, G.-M., Pfister, G.: Singular Introduction to Commutative Algebra. Extended. Springer, Berlin (2008)

10. Hampton, M., Jensen, A.: Finiteness of spatial central configurations in the fivebody problem. Celestial Mech. Dynam. Astronom. 109(4), 321-332 (2011). https://doi.org/10.1007/s10569-010-9328-9

11. Hept, K., Theobald, T.: Tropical bases by regular projections. Proc. Am. Math. Soc. 137(7), 2233-2241 (2009). https://doi.org/10.1090/S0002-9939-09-09843-8

12. Herrmann, S., Jensen, A., Joswig, M., Sturmfels, B.: How to draw tropical planes. Electron. J. Combin. 16(2), 1-26 (2009). Special volume in honor of Anders Björner, Research Paper 6

13. Herrmann, S., Joswig, M., Speyer, D.E.: Dressians, tropical Grassmannians, and their rays. Forum Math. 26(6), 1853-1881 (2014). https://doi.org/10.1515/forum-2012-0030

14. Hofmann, T., Ren, Y.: tropicalnewton.lib. A Singular 4-1-0 library for tropical computations using Newton polygon methods. http://www.singular.uni-kl.de. (2016)

15. Jensen, A.N: Gfan, a software system for Gröbner fans and tropical varieties. http://home.imf.au.dk/ jensen/software/gfan/gfan.html. (2017) 
16. Jensen, A., Ren, Y., Seelisch, F. : gfan.lib. A Singular 4-1-0 interface to gfanlib. http://www.singular. uni-kl.de. (2016)

17. Jensen, A., Sommars, J., Verschelde, J.: Computing Tropical Prevarieties in Parallel. In: Proceedings of the International Workshop on Parallel Symbolic Computation (PASCO'17), pp. 9:1-9:8. ACM, Kaiserslautern, Germany (2017). https://doi.org/10.1145/3115936.3115945

18. Jensen, A., Yu, J.: Stable intersections of tropical varieties. J. Algebraic Combin. 43(1), 101-128 (2016). https://doi.org/10.1007/s10801-015-0627-9

19. Lazard, D.: Gröbner bases, Gaussian elimination and resolution of systems of algebraic equations. In: van Hulzen, J.A. (ed.) Computer Algebra (London, 1983). Lecture Notes in Computer Science, vol. 162, pp. 146-156. Springer, Berlin (1983). https://doi.org/10.1007/3-540-12868-9_99

20. Lazard, D.: Solving zero-dimensional algebraic systems. J. Symb. Comput. 13(2), 117-131 (1992). https://doi.org/10.1016/S0747-7171(08)80086-7

21. Maclagan, D., Sturmfels, B.: Introduction to Tropical Geometry. Graduate Studies in Mathematics, vol. 161. American Mathematical Society, Providence, RI (2015)

22. Mikhalkin, G.: Enumerative tropical algebraic geometry in $\mathbb{R}^{2}$. J. Am. Math. Soc. 18(2), 313-377 (2005). https://doi.org/10.1090/S0894-0347-05-00477-7

23. Neukirch, J.: Algebraic Number Theory. Grundlehren der Mathematischen Wissenschaften, vol. 322. Springer, Berlin (1999). https://doi.org/10.1007/978-3-662-03983-0

24. Osserman, B., Payne, S.: Lifting tropical intersections. Doc. Math. J. DMV 18, 121-175 (2013)

25. Speyer, D., Sturmfels, B.: The tropical Grassmannian. Adv. Geom. 4(3), 389-411 (2004). https://doi. org/10.1515/advg.2004.023

26. Sturmfels, B.: Algebra. In: Pachter, L., Sturmfels, B. (eds.) Algebraic Statistics for Computational Biology, pp. 85-124. Cambridge University Press, New York (2005). https://doi.org/10.1017/ CBO9780511610684.006

27. Tran, N.M., Yu, J.: Product-mix auctions and tropical geometry. Math. Oper. Res. (2015). eprint: arXiv: 1505.05737 